\title{
Postpartum Daily Stress, Relationship Quality, and Depressive Symptoms
}

\author{
Melissa Page $\cdot$ Mari S. Wilhelm
}

Published online: 22 May 2008

(C) Springer Science+Business Media, LLC 2008

Erratum to: Contemp Fam Ther (2007) 29(4):237-251

DOI 10.1007/s10591-007-9043-1

Within the Results section, and beginning on page 245 at the end of the first paragraph, the following text was omitted from the original version of this article (Volume 29, Number 4, December 2007, pp. 237-251). We reproduce the text here as it should appear on page 245 in its entirety, with the omitted text highlighted in bold:

The result of the regression analysis is shown in Table 4. Depression during pregnancy had the strongest beta weight in both blocks, confirming previous findings for depression during pregnancy as a primary predictor of postpartum depression (Beck, 2001). Arguments with family reached significance $\beta=.300, \ldots P<.01)$ in the regression analysis, and was in the expected direction.

Relationship depth reached significance $(\beta=-.207, P<.05)$ in the regression analysis after controlling for depression during pregnancy and entering it simultaneously with arguments with family, and it too was in the expected direction. A third block in the regression analysis included an interaction (or moderator) between arguments with family and relationship depth. The presence of the moderator reduced the significance of relationship depth, but failed to reach significance $(P=.085)$, even though this additional block explained $3 \%$ more of the variance in postpartum depressive symptoms. The regression block including the moderator is not shown. Further, the interaction variable between support and arguments with family was tested in the model and was not significant.

The online version of the original article can be found under doi:10.1007/s10591-007-9043-1.

M. Page $(\square) \cdot$ M. S. Wilhelm

Family Studies and Human Development, University of Arizona,

FCS 217, P.O. Box 210033, Tucson, AZ 85721-0033, USA

e-mail: mpage@u.arizona.edu

M. S. Wilhelm

e-mail: wilhelmm@ag.arizona.edu 
A power analysis was conducted to determine the strength of the findings for such a small sample size. Using the procedure outlined in Cohen, Cohen, West, \& Aiken (2003), an $R^{2}=.561$ for the entire regression resulted in a power coefficient of 60.06 equivalent to a power level of .99 at $\alpha=.01$. A second power analysis was conducted to report on the variance attributed to arguments with family and relationship depth after removing the variance attributed to depression during pregnancy by using the change in $R^{2}$ from Step 1 to Step $2\left(R^{2} \Delta=.159\right)$. The power for this variance of two predictors equaled 8.89, which provides a power of .76 at $\alpha=.05$.

Post hoc analysis

Based on the postpartum depression literature, we decided to analyze and describe our nonclinical sample by utilizing the two cut-off points for the CES-D (16 and 24). We divided our sample into three groups: women scoring 0-15, women scoring 16-23, and women scoring 24-60. Table 5 includes the mean and standard deviation scores for the three subgroups, as well as $t$-test values located in the notes section. The sub-sample of 6 women scoring 16-23 represents $12 \%$ of the total sample $(n=51)$ and $40 \%$ of the depressed sample $(n=15)$. The sub-sample of 9 women scoring 24 or above represents $18 \%$ of the total sample $(n=51)$ and $60 \%$ of the depressed sub-sample $(n=15)$.

A $T$-test comparison of women scoring 0-15 and 16-23 on the CES-D resulted in two variables that were significantly different (postpartum depression, $t=-6.261, P<.001$; and relationship support, $t=3.48, P<.01)$.

The second $T$-test compared women scoring 0-15 with those scoring 24-60 and resulted in seven variables reaching significance (postpartum depression, $t=-11.413, P<.001$, depression during pregnancy, $t=-4.014, P<.001$, educational attainment, $t=3.222$, $P<.01$, demands from home, $t=-2.352, P<.05$, arguments with family, $t=-2.631$, $P<.05$, relationship support, $t=3.804, P<.001$, relationship depth, $t=3.456, P<.01$ ). A third comparison was completed for women scoring 16-23 with those scoring greater than 24; the only variable significantly different between these two groups was the level of postpartum depression $(t=-3.147, P<.01)$. 